\title{
Inhibition of Lipoprotein Lipase by Plasma from Children with the Steroid Responsive Nephrotic Syndrome
}

\author{
C. VERMYLEN, M. LEVIN, T. M. BARRATT, AND D. P. R. MULLER \\ Department of Paediatric Nephrology and Department of Child Health [D.P.R.M.], Institute of Child Health, \\ London, England
}

\begin{abstract}
The study was designed to investigate the hyperlipidaemia associated with the steroid responsive nephrotic syndrome in children and in particular to examine the mechanism for the delayed clearance of the circulating triglyceride-rich lipoproteins. The possibility that plasma from patients with steroid responsive nephrotic syndrome may contain an inhibitor of lipoprotein lipase activity was studied by examining the effect of the addition of plasma from patients, on normal postheparin lipoprotein lipase activity. Plasma from children with steroid responsive nephrotic syndrome significantly inhibited lipoprotein lipase activity $(p<0.001)$, whereas that from patients with familial hypercholesterolaemia and normal children had no significant effect. The inhibition of lipoprotein lipase activity by plasma from patients with steroid responsive nephrotic syndrome correlated significantly with their increased plasma cholesterol and reduced plasma albumin concentrations $(p<0.001$ and $<0.02$, respectively), but there was no significant correlation with plasma triglyceride concentrations. Thus, the degree of inhibition probably reflected the severity of the condition at the time of study. Neither the cholesterol, albumin nor triglyceride concentrations appeared to directly influence the lipoprotein lipase activity of postheparin plasma. (Pediatr Res 22: 197200, 1987)
\end{abstract}

Abbreviation

SRNS, steroid responsive nephrotic syndrome

Hyperlipidaemia is a characteristic feature of SRNS, with untreated patients having increased plasma concentrations of cholesterol and triglyceride, and lipoprotein carriers, chylomicrons, very low density, intermediate density, and low density lipoproteins (1-3). Studies on the mechanism of the hyperlipidemia suggest that there is both an increased synthesis and secretion of the lipoproteins (4-7) as well as a reduced clearance of circulating triglyceride (4-8).

Triglycerides are transported in the circulation by the triglyceride-rich lipoproteins (chylomicrons and very low density lipoproteins) which are cleared from the circulation by the action of lipoprotein lipase. This enzyme is located on the capillary endothelium of extrahepatic tissues but can be released into the circulation by low doses of intravenous heparin. Postheparin

Received September 16, 1986; accepted March 18, 1987

Correspondence Dr. D. P. R. Muller, Department of Child Health, Institute of Child Health, 30 Guilford Street, London WCIN 1EH, England.

Supported by the Catholic University of Louvain, Belgium and the Kidney Reseach Aid Fund, UK. plasma also contains a lipase of hepatic origin which has different properties to lipoprotein lipase (9).

We have previously studied the activities of lipoprotein lipase and hepatic lipase in postheparin plasma from patients with secondary hypertriglyceridemia as a result of uncontrolled glycogen storage disease $(10,11)$. These patients had greatly reduced activities of both lipoprotein and hepatic lipase which resulted from the presence of a circulating inhibitor (11).

Reduced activities of lipoprotein lipase and hepatic lipase have been previously reported in patients with SRNS (12) and rats with experimental nephrosis (7) but the mechanism has not been established. Reduced enzymatic activities could result from a quantitative reduction in enzyme protein, an inhibition of enzymatic activity or an abnormal interaction between heparin and the lipase at the capillary endothelium. In this study we investigated the possibility that the reduced enzymatic activity may result from the presence of a circulating inhibitor.

\section{PATIENTS AND METHODS}

Patients. Fourteen children (10 males and four females) aged 3-16 yr with SRNS were studied. Five had minimal change histology and in the remainder this was presumed on the basis of steroid response. They were in relapse $(3+$ proteinuria using Albustix) and were not receiving steroid therapy at the time of study. Five patients (four male and one female) aged 6-16 yr with familial hypercholesterolemia were studied while on a fat modified diet or receiving cholestyramine (Bristol Laboratories, Slough, England). The diagnosis was based on standard criteria (13). A control group of 12 children (10 males and two females) aged 12-14 yr was studied. These children were part of the Chard study of normal growth and adolescence which was approved by the Ethical Committee of the Hospitals for Sick Children, London.

Methods. Postheparin Plasma. Postheparin plasma was obtained from a healthy adult by venepuncture exactly $10 \mathrm{~min}$ after an intravenous injection of heparin (10 U/ $\mathrm{kg}$ body weight). Blood was collected into glass tubes containing dipotassium EDTA (1.5 $\mathrm{mg} / \mathrm{ml}$ ) and the postheparin plasma was stored in aliquots at $-20^{\circ} \mathrm{C}$ and used throughout as source of normal lipoprotein lipase activity.

Inhibitor Studies. Blood was collected from the children under investigation into $3.8 \%$ sodium citrate $(1: 10 \mathrm{v} / \mathrm{v})$. The plasma was separated and stored at $-70^{\circ} \mathrm{C}$ prior to assay. The inhibition of normal lipoprotein lipase activity was estimated by mixing 50 $\mu \mathrm{l}$ of the test plasma with $50 \mu \mathrm{l}$ of the normal post-heparin plasma and assaying the lipoprotein lipase activity essentially as described previously using tritiated $\left(9,10-{ }^{3} \mathrm{H}\right)$ glycerol trioleate as substrate (10). A final concentration of sodium chloride of 0.075 $\mathrm{mol} / \mathrm{liter}$ was used throughout and aliquots of the reaction mixture were removed at 40,55 , and $70 \mathrm{~min}$. The radiolabeled 
fatty acids released by lipolytic activity were extracted and counted on an LKB Wallac Ultrabeta liquid scintillation counter using external standardisation for quench correction. Addition of plasma from normal children gave the expected activity with no significant inhibition. A pool of plasma samples from normal children was therefore used as a quality control in each batch of assays and assigned an activity of $100 \%$. The results of the test samples were expressed as a percentage of this quality control value.

Miscellaneous Methods. Plasma triglyceride and cholesterol concentrations were measured enzymatically using commerical kits supplied by Metachem Diagnostics and Abbott Laboratories Limited, respectively. Serum albumin concentrations were estimated by single radial gel diffusion (14).

Statistics. Results are expressed throughout as mean $\pm 1 \mathrm{SD}$ and the significance of differences between mean values was calculated by the Student's $t$ test. Correlation coefficients were derived from linear regression analysis.

\section{RESULTS}

The fasting plasma triglyceride, cholesterol, and albumin concentrations in the children with SRNS and familial hypercholesterolemia and the control children are shown in Table 1. The children with SRNS had significantly increased fasting triglyceride and cholesterol concentrations $(p<0.02$ and $<0.001$, respectively) and a significantly reduced plasma albumin concentration $(p<0.01)$ compared to the controls. The children with familial hypercholesterolemia had a significantly raised plasma cholesterol concentration $(p<0.001)$ which was not significantly different from that observed in SRNS.

The effect of adding plasma from patients in the various clinical groups to a normal postheparin plasma sample is shown in Figure 1. Results are expressed as the percentage of the lipoprotein lipase activity obtained with the quality control specimen. The activities obtained with the plasmas from the 12 control children were distributed around the quality control value $(104.6 \pm 10.5 \%)$. Plasma from the children with SRNS showed a significant inhibition $(p<0.001)$ of the lipoprotein lipase activity $(68.4 \pm 22.0 \%)$ whereas that from the patients with familial hypercholesterolemia did not differ significantly from the control values $(91.7 \pm 14.0 \%)$.

The percentage of the quality control lipolytic activity obtained with the SRNS samples was correlated with the plasma lipid and albumin concentrations (Fig. 2). A significant positive correlation $(p<0.02)$ was found with the albumin concentration and a significant negative correlation $(p<0.001)$ was observed with plasma cholesterol concentrations but there was no significant correlation with plasma triglyceride concentrations.

To investigate whether the inhibition of lipoprotein lipase activity resulted directly from either the reduced albumin or increased triglyceride concentrations, increasing amounts of albumin were added to a plasma sample from a patient with SRNS and increasing amounts of triglyceride in the form of Intralipid (Kabi Vitrum, UK) to three control plasma samples. The final concentrations of albumin and triglyceride ranged from 10 to 45 $\mathrm{g} /$ liter and 1 to $15 \mathrm{mmol} /$ liter, respectively. The highest triglyceride concentrations (15 mmol/liter) tended to give a slight reduction of lipoprotein lipase activity ( $80 \%$ of starting activity) (see Fig. 3) but otherwise there was no effect on lipolytic activity.

\section{DISCUSSION}

We have previously observed that patients with SRNS show a decreased sensitivity to the anticoagulant effect of heparin (15). The release of lipoprotein and hepatic lipase from the capillary endothelium into the blood stream by heparin may therefore also be impaired in SRNS, resulting in reduced activities of these enzymes in postheparin plasma from patients with this condition. For this reason we decided not to estimate postheparin lipolytic activity directly but to investigate the possible inhibitory activity of plasma from children with SRNS by adding it to normal postheparin plasma and assaying the lipoprotein lipase activity. Using this approach we observed a highly significant inhibition of lipoprotein lipase activity with plasma samples from children with SRNS, whereas plasma from patients with familial hypercholesterolemia or normal controls showed no inhibition. The range of inhibition observed in SRNS was wide and probably reflected variation in the severity of the condition at the time of

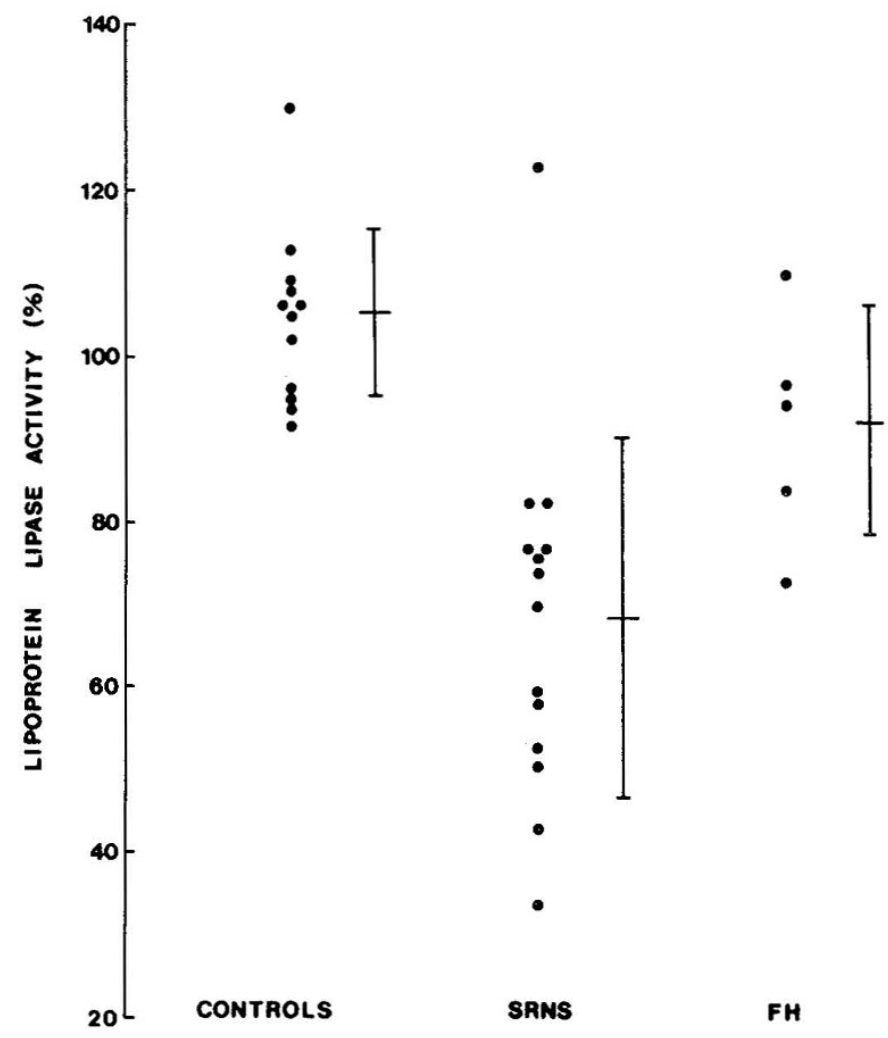

Fig. 1. The effect of adding plasma from control children and children with SRNS and familial hypercholesterolemia $(F H)$ to normal postheparin plasma. Lipoprotein lipase activity is expressed as the percentage of the value obtained with the quality control (plasma from a group of normal children) taken as $100 \%$. The bars denote mean \pm 1 SD.

Table 1. Fasting plasma lipid and albumin concentrations in the patient groups

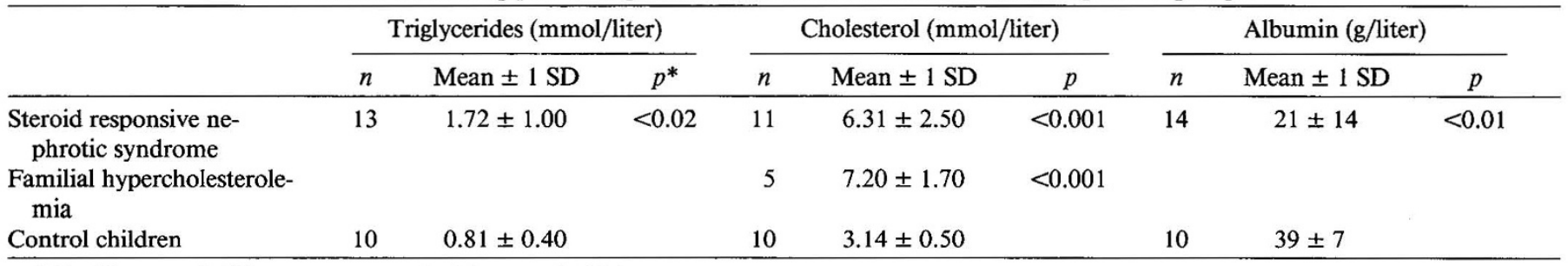

* Significance of difference compared with control children. 


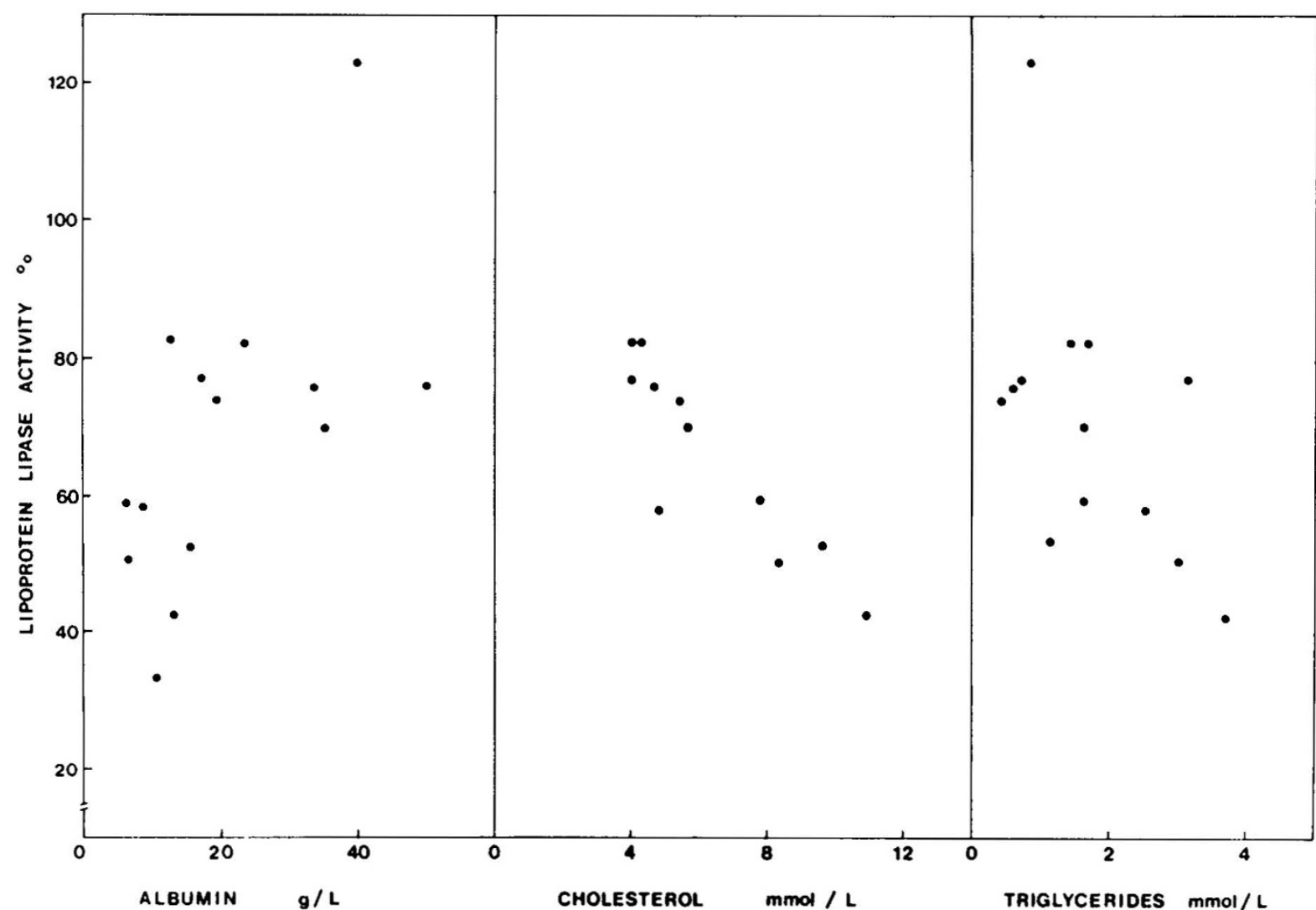

Fig. 2. Correlation of lipoprotein lipase activity (expressed as a percentage of the value obtained with the quality control taken as $100 \%$ ) with the plasma concentrations of albumin $(p<0.02)$, cholesterol $(p<0.001)$, and triglyceride (not significant).

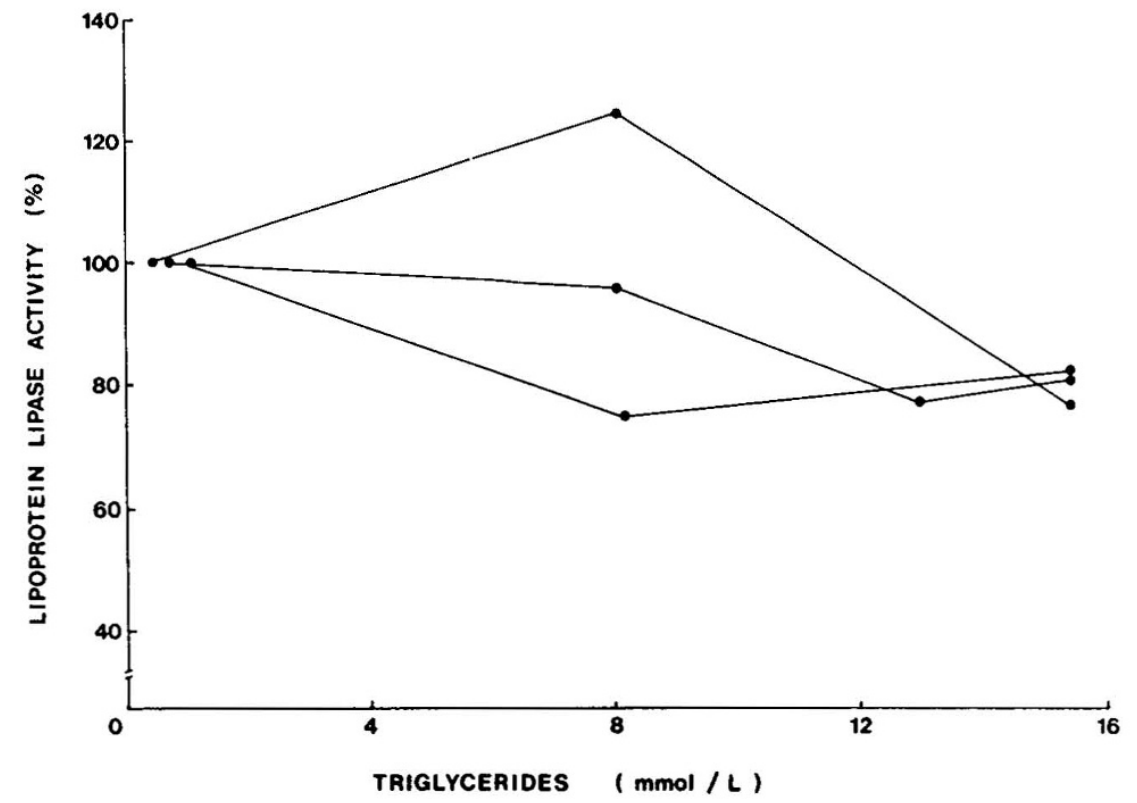

Fig. 3. The effect of adding increasing amounts of triglyceride in the form of Intralipid to three normal plasmas. The plasmas were then added to a normal postheparin plasma. The lipoprotein lipase activity is expressed as a percentage of the activity obtained with no added Intralipid which is taken as $100 \%$.

study. This was suggested by the significant correlation of increasing inhibition of lipolytic activity with decreasing plasma albumin and increasing cholesterol concentrations.

The nature of the inhibitor is not known. It does not appear to be a consequence of increased substrate concentration as increasing the triglyceride concentration in vitro over the range found in the plasma specimens did not inhibit the enzyme activity. We have also previously shown that postheparin plasma 
from a patient with type I hyperlipoproteinemia and a triglyceride concentrations of $7 \mathrm{mmol} /$ liter was not inhibitory (11). Similarly the addition of albumin to nephrotic plasma did not significantly alter the lipoprotein lipase activity. This is in agreement with the observation of Marsh (6) that the intravenous injection of albumin into rats with experimental nephrosis did not correct the catabolic defect, although others have reported that albumin can reverse the hyperlipemia in patients with the nephrotic syndrome (16).

It has been reported that increased cholesterol concentrations can inhibit lipoprotein lipase activity (17). In our study, however, plasma cholesterol concentrations were similar in the patients with SRNS and familial hypercholesterolemia, and plasma from the latter group of patients did not show significant inhibition. Lipoprotein lipase requires a cofactor, apoprotein CII, for activity but this apoprotein is present in large amounts in patients with SRNS (6), and the absence of an activator is unlikely to account for the observed inhibition of normal lipoprotein lipase activity.

There is evidence that proteinuria in SRNS is caused by a loss of negative charge on the glomerulus (18). A loss of surface charge on red blood cells and platelets has also been reported (19) and this together with the reduced sensitivity to heparin in SRNS suggests the presence of a circulating cationic macromolecule. The site of action and the activity of lipoprotein lipase are effected by charged macromolecules. Thus lipoprotein lipase binds to negatively charged glycosaminoglycans (probably heparan sulphate) at the capillary endothelium via a specific binding site $(20)$. The enzyme is inhibited by protamine and there is evidence that it can be activated in vitro by heparan sulfate (21). It is therefore possible that the presence of a circulating cationic macromolecule may be responsible for the inhibition of lipoprotein lipase activity in SRNS.

We have previously documented the presence of a circulating inhibitor of lipoprotein lipase and hepatic lipase activity in patients with glycogen storage disease (11) and preliminary results suggest that plasma from children with uncontrolled insulindependent diabetes mellitus is also inhibitory. It therefore appears that a number of secondary hyperlipidemic conditions may result at least in part from the presence of circulating inhibitor(s) of lipoprotein lipase activity with a consequent decrease in the rate of clearance of the triglyceride-rich lipoproteins.

Acknowledgments. The authors thank Professor M. Preece (Department of Growth and Development, Institute of Child Health) and the children of the Chard Study for their help in obtaining the control data.

\section{REFERENCES}

1. Baxter JH, Goodman HC, Havel RJ 1960 Serum lipid and lipoprotein alterations in nephrosis. J Clin Invest 39:455-456

2. Moorhead JF, El-Nahas M, Chan MK, Varghese Z 1982 Lipid nephrotoxicity in chronic progressive glomerular and tubulointerstitial disease. Lance 2:1309-1311

3. Appel GB, Blum CB, Chien S, Kunis CL, Appel AS 1985 The hyperlipidemia of the nephrotic syndrome. Relation to plasma albumin concentration, oncotic pressure and viscocity. N Engl J Med 312:1544-1548

4. Kekki M, Nikkila EA 1971 Plasma triglyceride metabolism in nephrotic syndrome. Eur J Clin Invest 1:345-351

5. Chan MK, Persaud JW, Ramdial L, Varghese Z, Sweny P, Moorhead JF 1981 Hyperlipidaemia in untreated nephrotic syndrome, increased production or decreased removal? Clin Chim Acta 117:317-323

6. Marsh JB 1984 Lipoprotein metabolism in experimental nephrosis. J Lipid Res 25:1619-1623

7. Garber DW, Gottleib BA, Marsh JB, Sparks CE 1984 Catabolism of very low density lipoproteins in experimental nephrosis. J Clin Invest 74:1375-1383

8. Gitlin D, Cornwell DG, Makasato D, Oncley JL, Hughes WL, Janeway CA 1958 Studies on the metabolism of plasma proteins in the nephrotic syndrome II. The lipoproteins. J Clin Invest 37:172-184

9. La Rosa JC, Levy RI, Windmueller HG, Fredrickson DS 1972 Comparison of the triglyceride lipase of liver, adipose tissue and post-heparin plasma. $J$ Lipid Res 13:356-363

10. Gamlen TR, Muller DPR 1980 The validation and use of specific methods for the estimation of lipoprotein lipase and hepatic lipase activities in postheparin plasma of children with hyperlipidaemia. Clin Chim Acta 106:7583

11. Muller DPR, Gamlen TR 1984 The activity of hepatic lipase and lipoprotein lipase in glycogen storage disease: evidence for a circulating inhibitor of postheparin lipolytic activity. Pediatr Res 18:881-885

12. Yamada K, Matsuda J 1970 Lipoprotein lipase in clinical and experimental nephrosis. Clin Chim Acta 30:787-794

13. Fredrickson DS, Levy RI 1972 Familial hyperlipoproteinaemia. In: Stanbury JB, Wyngaarden JB, Fredrickson DS (eds) The Inherited Basis of Metabolic Disease. McGraw-Hill, New York, pp 545-614

14. Mancini G, Carbonara AO, Heremans JF 1965 Immunochemical quantification of antigens by single radial immunodiffusion. Int $J$ Immunochem 2:235254

15. Vermylen CG, Levin M, Lanham JG, Hardisty RM, Barratt TM Decreased sensitivity to heparin in vitro in steroid responsive nephrotic syndrome. Kidney Int 31:1396-1401

16. Cohen SL, Cramp DG, Lewis AD, Tickner TR 1980 The mechanism of hyperlipdaemia in nephrotic syndrome-role of low albumin and the LCAT reaction. Clin Chim Acta 104:393-400

17. Fielding CJ 1970 Human lipoprotein lipase inhibition of activity by cholesterol. Biochim Biophys Acta 218:221-226

18. Robson AM, Giangiacomo J, Kienstra RA, Naquvi ST, Ingelfinger JR 1974 Normal glomerular permeability and its modification by minimal change nephrotic syndrome. J Clin Invest 54:1190-1199

19. Levin M, Smith C, Walters MDS, Gascoine P, Barratt TM 1985 Steroid responsive nephrotic syndrome: a generalised disorder of membrane negative charge. Lancet 2:239-242

20. Olivecrona T, Bengtsson G, Marklund S-E, Lindahl U, Hook M 1977 Heparinlipoprotein lipase interactions. Fed Proc 36:60-65

21. Staprans I, Felts JM 1985 Isolation and characterization of glycosaminoglycans in human plasma. 76:1984-1991 